\title{
Enhancement of interfacial adhesion in glass fiber/epoxy composites by electrophoretic deposition of graphene oxide on glass fibers
}

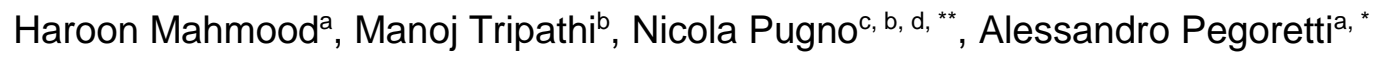 \\ a Department of Industrial Engineering and INSTM Research Unit, University of Trento, via \\ Sommarive 9, 38123 Trento, Italy \\ b Centre for Materials and Microsystems, Fondazione Bruno Kessler, via Sommarive 18, I-38123 \\ Povo (Trento), Italy \\ ${ }^{c}$ Laboratory of Bio-Inspired and Graphene Nanomechanics, Department of Civil, Environmental and \\ Mechanical Engineering, University of Trento, via Mesiano 77, I-38123 Trento, Italy \\ d School of Engineering and Materials Science, Queen Mary University of London, Mile End Road, \\ E1 4NS London, UK
}

\begin{abstract}
Graphene oxide (GO) can increase the fiber/matrix interfacial shear strength (ISS) in glass fibers (GF) reinforced epoxy composites. To validate our argument, GO was synthesized and deposited over GF through electrophoretic deposition. Tuned voltage lead to different thickness of deposited GO steadily increasing with the electric field up to $10 \mathrm{~V} / \mathrm{cm}$. Coated fibers were aligned in a mold and an epoxy matrix was used to create a single-fiber microcomposite. Fragmentation test showed significantly higher ISS values for coated over bare fibers with increments up to a factor of about 2 and proportional to the amount of GO deposited on the GF. Tribological tests were performed at nanoscale by atomic force microscopy to measure the delamination strength between GO and GF. This latter resulted to be much higher than the ISS thus proving the efficiency of the new GO fiber coating method here proposed for producing advanced graphene based composites. The failure of the composite at the GO/matrix rather than at the GO/GF interface was also confirmed by scanning electron microscopy observation of the fracture surfaces of microcomposites.
\end{abstract}

\section{Keywords}

Nano composites, Fiber/matrix bond, Fragmentation, Interfacial strength, Delamination, Graphene

\section{Introduction}

The use of composite materials has grown in almost every engineering sector over the past 35 years. This increasing interest is mostly due to the unique properties of polymer composites in terms of lightness, rigidity, strength, easy formability, damage tolerance and corrosion resistance. Notably, there is an enormous potential for growth on the global market since both advanced and commodity composites still only own a relatively small market percentage in contrast to competing materials such as steel and aluminum [1]. Properties of composite materials are largely determined by the fiber/matrix interfacial adhesion [2,3]. In fact, an effective interface is necessary to guarantee the stress transfer from the matrix to the load-bearing fibers. The fiber-matrix interface transfers stresses via combination of mechanical interlocking, chemical bonding and physical adhesion [4]. However, due to limitations such as the lack of reactive functional groups on the fiber surface and poor wettability, effective transfer of load between fibers and matrix is a difficult task. Hence it has been of prime concern in the research areas of both academia and industries to enhance the interfacial adhesion between fiber and matrix. 
Adhesion strength between fibers and matrices can be improved by various approaches. As described in a recent review paper by Karger-Kocsis et al. [5], the most common methods can be classified on the basis of the selected strategies distinguishing between i) interphase tailoring via sizing/coating on fibers, ii) creation of hierarchical fibers by nanostructures, iii) fiber surface modifications by polymer deposition and iv) potential effects of matrix modifications on the interphase formation. In particular, development of hierarchical micro/nano composites and its theoretical investigation [6] has been explored with great interest in recent years, specifically on the integration of nanoscale reinforcement on fibers [7,8]. Growth or deposition of carbon nanotubes (CNTs) on reinforcements for better interfacial adhesion has been reported in numerous articles. The use of chemical vapor deposition (CVD) [9], grafting technique [10,11], electrophoretic deposition (EPD) [12] has been successfully implemented for the growth/deposition of nanomaterials on various fibers. Wang et al. used multi-walled carbon nanotubes (MWCNTs) as an interphase between GF and vinyl ester composites. They reported improved bonding between the GF and the resin matrix [13]. Similarly Zhang et al. found an increase of $30 \%$ ISS by EPD to deposit MWCNTs on the surface of GF [14]. The electrical conductivity of fibers also improved due to deposited MWCNT. Exfoliated graphite nanoplatelets were deposited by Park et al. [15] using EPD onto single carbon fibers.

Graphene, being a superlative nanomaterial, has been considered to be a promising and exciting research area [16] because of its outstanding ideal properties including electron mobility at room temperature $(250000 \mathrm{~cm} 2 / \mathrm{V})$, thermal conductivity $(5000 \mathrm{~W} \mathrm{~m}-1 \mathrm{~K} 1$ ) and mechanical properties, with a Young's modulus of $1 \mathrm{TPa}$ and a record-breaking strength of $130 \mathrm{GPa}$ [17e19]. Hu et al. have recently discussed in detail the use of graphene polymer nanocomposites for structural and functional applications [20]. The incorporation of graphene in polymer based composites has resulted in improved performance due to the interfacial interactions between polymers and graphene-based materials [21e23]. It has been reported that with polymers, the molecular interactions of graphene consist of either hydrophobichydrophobic interactions, weak van der Waal's forces and p-p stacking [24e28]. The use of oxidized form of graphene in polymer matrix gives more versatility of interactions due to the presence of oxygen-containing polar functionalities like carboxyl, carbonyl, epoxide and hydroxyl groups [29]. The elastic modulus of the polymer composites increases greatly due to the interfacial crosslinking [30] whereas electrostatic interactions are also important which result in nanocomposites much stronger and tougher [31].

This research group previously evaluated the role of graphene nanoplatelets in GF/epoxy matrix composite either by sonication in the epoxy matrix or dip coating the fibers in graphene dispersion [32]. An increase of elastic modulus, without compromising the impact strength and with an enhancement of the viscoelastic properties of composites, was reported.

This current work was aimed to assess the interfacial shear strength between an epoxy matrix and GFs coated with electrophoretically deposited graphene oxide. In addition, atomic force microscope (AFM) tribological studies were performed using diamond probe. Delamination of GO was performed with optimized normal force to calculate the shear strength of the GO/GF interface.

\section{Experimental section}

\subsection{Materials and samples preparation}

All chemicals were of analytical grade and used without further purification. Graphite powder, sodium nitrate, potassium permanganate, sulfuric acid and hydrogen peroxide were purchased from Sigma Aldrich while hydrochloric acid was from Codec Chemical Co. Ltd.. E-glass fibers (manufactured by PPG, trade name: 2001 ) having a diameter of $25.1 \pm 0.4 \mathrm{~mm}$ were used as reinforcement. This GF had an epoxy-compatible sizing and it was used as received. A bicomponent epoxy resin (epoxy base EC 252 and hardener W 241) was provided by Elantas Italia S.r.I. The physical properties of epoxy resin cured at room temperature for $3 \mathrm{~h}$ followed by $15 \mathrm{~h}$ at $60 \mathrm{C}$ are summarized in Table 1 . 
Graphene oxide was synthesized using an approach similar to Hummer's method [33]. Briefly, $1 \mathrm{~g}$ graphite powder was added into $46 \mathrm{ml}$ of $\mathrm{H} 2 \mathrm{SO} 4$ cooled in an ice bath, followed by the addition of 1 $\mathrm{g}$ of $\mathrm{NaNO} 3$ and stirred for $15 \mathrm{~min}$. In the next step, $6 \mathrm{~g}$ of $\mathrm{KMnO} 4$ were slowly added in order to avoid a spontaneous exothermic reaction. The mixture was then stirred for at least $24 \mathrm{~h}$ at $35 \mathrm{C}$. Finally, excess of distilled water was added to the above mixture while the temperature was kept under $80 \mathrm{C}$. In the end, $30 \% \mathrm{H} 2 \mathrm{O} 2$ was added to the mixture to stop the reaction. The resulting suspension was thoroughly washed using $\mathrm{HCl}$ solution and distilled water to remove $\mathrm{Mn}$ ions and acid respectively. The obtained brown solution was dried in a vacuum oven at $50 \mathrm{C}$ for at least $36 \mathrm{~h}$.

A schematic description of the EPD process used to deposit GO nanosheets on GFs is depicted in Fig. 1. A stable suspension is the key for uniform deposition of graphene on GFs. Initially, graphite oxide powder was added in water with a concentration level of $1 \mathrm{mg} / \mathrm{ml}$ and the dispersion was subjected to bath-sonication for $1 \mathrm{~h}$. Since GFs are non-conductive materials, two copper plates were used as electrodes in the EPD process. The GFs (fixed on a window frame) were placed near the anode since GO display negative potential due to functionalities attached during the oxidation reaction. Hence, during the EPD process GO migrated towards the anode and deposited on the GFs. EPD was carried out at various applied voltages up to $10 \mathrm{~V} / \mathrm{cm}$ with a constant deposition time of $5 \mathrm{~min}$ and electrodes gap of $2 \mathrm{~cm}$. A second EPD cycle was performed under the same conditions while reversing the GFs so that a homogenous deposition could be achieved on the fiber surface. The coated samples were dried in a vacuum oven at $40 \mathrm{C}$ for $12 \mathrm{~h}$.

\subsection{Testing methods}

The morphology of GO nanosheets coatings were investigated by field emission scanning electron microscopy (FESEM) by a Zeiss SUPRA 40 microscope. Approximately $5 \mathrm{~nm}$ thick layer of platinum was deposited on samples prior to FESEM observations. Thickness (z-direction) and roughness of GO coatings on GF were measured by AFM with a NT-MDT solver P47h device operated in intermittent contact mode (tapping mode).

The oxidation level of graphite was evaluated using X-ray Tab diffraction technique by a Rigaku III D-max diffractometer (monochromatic radiation CueKa line with I = 51.54056 ̊̊). Measurements were carried out in the $2 q$ range of $5 \mathrm{e} 80$ with a step size of 0.04 .

A Nikolet Avatar 330 device with a $4 \mathrm{~cm} 1$ resolution was used to record Fourier transform infrared (FTIR) spectra. The graphite powder and graphene oxide powder were individually mixed with potassium bromide $(\mathrm{KBr})$ powder to form homogeneous mixtures and thin discs for analysis were made in a compression mold at 10 bar pressure.

Differential scanning calorimetry (DSC) analyses were performed by using a Mettler DSC 30 calorimeter. The experiments were performed at a heating rate of $10 \mathrm{C} / \mathrm{min}$ and a constant nitrogen flux of $100 \mathrm{~mL} / \mathrm{min}$ was maintained during the tests in the temperature range from 0 to $80 \mathrm{C}$.

Thermogravimetric analysis (TGA) was performed with a Mettler TG 50 thermobalance at a heating rate of $10 \mathrm{C} / \mathrm{min}$ and $100 \mathrm{~mL} / \mathrm{min}$ of nitrogen flux during the test. The temperature range was $25 \mathrm{e} 750$ C.

Uniaxial tensile tests of the epoxy polymer were performed with an Instron® 5969 universal testing machine. A sample of at least five ISO 527 type 1BA specimens were prepared and the tests were carried out at a crosshead speed of $10 \mathrm{~mm}$ min 1 up to $1 \%$ axial deformation. The elastic modulus was measured as a secant value between deformation levels of 0.05 and $0.25 \%$ as per ISO 527 standard.

A tensile tester (Minimat, by Polymer Laboratories, Loughborough, UK) was used to perform the single-fiber fragmentation tests (SFFT). The tests were performed at ambient temperature while a polarized optical stereo-microscope (Wild M3Z by Leica) was used to observe the fiber fragmentation process during the tensile test. A cross-head speed of $10 \mathrm{~mm} / \mathrm{min}$ was applied up to a strain of $10 \%$, 
necessary to assure the saturation of the fragmentation process. The mean fiber length at saturation, LS, was measured by an image analysis software (Image J). The value of critical fiber length, LC, was considered to be equal to 0.75 LS. ISS values were derived according to the simplified micromechanical model proposed by Kelly and Tyson [34]. The static equilibrium between the tensile force acting on a fiber and the shear force transferred through the fiberematrix interface results in an average value of ISS according to the following equation:

$$
I S S=\frac{\sigma_{\mathrm{fb}}\left(\mathrm{L}_{\mathrm{c}}\right) \mathrm{d}}{2 \mathrm{~L}_{\mathrm{c}}}
$$

where $d$ is the fiber diameter and sfbðLcP is the tensile strength of a fiber at the critical length. This latter value can be estimated by assuming a Weibull distribution for the fiber strength, i.e.:

$$
\sigma_{\mathrm{fb}}\left(\mathrm{L}_{\mathrm{c}}\right)=\sigma_{0}\left(\frac{\mathrm{L}_{\mathrm{c}}}{\mathrm{L}_{0}}\right)^{\frac{1}{\mathrm{~m}}} \Gamma\left(1+\frac{1}{\mathrm{~m}}\right)
$$

where $\mathrm{G}$ is the Gamma function, whereas $\mathrm{s} 0$ and $\mathrm{m}$ are the scale and shape parameters of the Weibull strength distribution at the reference length $L 0$, respectively. These parameters were estimated from strength data determined at one single gauge length by fitting the distribution of failure probability. In particular, single filaments of fiber were tested according to the ASTM standard C1557-03. Specimens were tested with an Instron 4502 universal tensile tester equipped with a 10 $\mathrm{N}$ load cell. A gauge length of $20 \mathrm{~mm}$ was adopted and the cross-head speed was fixed at 0.2 $\mathrm{mm} / \mathrm{min}$. An iterative procedure originally proposed by Gurvich et al. [35] was used for the data reduction whose outcome is summarized in Table 2.

FFM (friction force microscopy) was conducted in contact mode (in AFM set-up) using diamond coated cantilever tip apex (model: DCP01_NTMDT). Here, AFM plays a dual role for mapping and manipulation the substrate sequential manner. Sader method $[36,37]$ was applied to measure normal $(\mathrm{KN})$ and torsional (KT) spring constants of cantilever. Typical values of KN z $6.03108 \mathrm{~N} / \mathrm{m}$ and KT z $8.25108 \mathrm{~N} / \mathrm{m}$ for cantilever with tip radius of $51 \mathrm{~nm}$ were obtained. Calibrated tip was slid from bare GF to GO covered region at a fixed normal force (FN). This method is capable of measuring substrate-coating adhesion energy by debonding the deposited GO. For an accurate estimation of adhesion force, the value of lateral force (FL) on bare substrate (here GF) was subtracted from the FL curve obtained by delaminating GO. The area under the curve is calculated to estimate the adhesion energy of the GO. Therefore, the FL required to delaminate GO from GF is defined as the adhesion of GO over GF and this value is calculated as work of adhesion [38]. Accordingly, FN $1 / 4$ $3.7 \mathrm{mN}$ was the minimal value to peel GO off the GF substrate.

\section{Results and discussion}

\subsection{Electrophoretic deposition process}

Fig. 2 shows the FESEM picture of an exfoliated sheet of GO. The sheet is several micrometers in lateral size. Wrinkling of GO sheet is also visible, which is basically a common phenomenon in two dimensional films and membranes.

Fig. 3a, 3b, 3c, 3d and 3e show an overview of the surfaces of uncoated and GO coated GFs. When the deposition voltage is increased from $2.5 \mathrm{~V} / \mathrm{cm}$ to $10 \mathrm{~V} / \mathrm{cm}$, the GO coating deposited on GFs appears to be more and more thick and uniform.

The amount of GO deposited on the GFs was estimated by weighing the GF bundle before and after the EPD process. The rate of weight increase of the GF bundle is reported in Fig. 4 as a function of the deposition voltage. It is worthwhile to note that a linear correlation between the deposition rate 
and the intensity of the electrical field can be observed. The linear fit of average values (line in Fig. 4) indicates a rate of weight increases of GO on GFs of $0.00133 \pm 0.00003 \% \mathrm{~cm} \min 1 \mathrm{~V} 1$. A similar trend was observed also by An et al. [39] who studied the EPD process of CNTs onto Eglass fibers fabrics. In fact, a mass change of the E-glass fabric linearly increasing with the applied electrical field used for the EPD of CNTs was reported.

\subsection{XRD and FTIR analysis}

XRD was employed to characterize the pristine graphite and the synthesized GO nanoparticles. The obtained XRD patterns are reported in Fig. 5. Graphite has a diffraction peak located at 2q 1/4 26.5 corresponding to the diffraction of (002) plane which indicates a highly organized crystallized structure. On the other hand, graphite oxide diffractogram (002) demonstrates a peak at 2q $1 / 410.9$ with relatively less intense and broad peak. Hence the oxidation of graphite led to the shift of diffraction peak position from 26.5 to 10.9 . The shift of diffraction peak is basically caused by the functional groups of oxygen on the sides of single graphene layer during Fi its oxidation [40].

FTIR spectra of graphite and GO are reported in Fig. 6. GO shows an increased amount of epoxy $\mathrm{CeOeC}$ groups at $1085 \mathrm{~cm} 1, \mathrm{C}] \mathrm{O}$ groups at $1625 \mathrm{~cm} 1$ and OeH group at $3830 \mathrm{~cm} 1$ thus confirming the increased level of oxidation of graphite when $\mathrm{GO}$ is produced.

\subsection{Single fiber fragmentation test}

The values of fragment length measured in SFFT and subsequent calculations of ISS values are given in Table 3. The length of fragments at saturation decreases as the deposition voltage increases which corresponds to an increasing thickness of GO coating. Interestingly, the ISS values increase in proportion as seen in Fig. 7. A remarkable improvement of $219 \%$ of ISS can be observed in case of GF coated with GO electrophoretically deposited at $10 \mathrm{~V} / \mathrm{cm}$. This positive effect could be tentatively attributed to the fact that GO creates a favorable bond between the GFs and epoxy resin which ultimately enhances the effective distribution of load on the GF. In such condition, the mechanical interlocking due to an increased surface roughness and the good adhesive compatibility between the epoxy matrix and GO are both responsible for the observed increase of ISS in microcomposites [41]. The steadily increment of the ISS values with the deposition voltage indicate that the ISS is related to the thickness of the GO layers deposited on the GF. This experimental evidence could be explained by the good efficiency of the load transfer mechanisms due to the surface functional groups available on the GO layers and the concurrent increase of the apparent fiber diameter due to the GO coating. A FESEM analysis of the GF protruding out the fracture surface of a microcomposite specimen is reported in Fig. 3f. It is interesting to observe how the GF fiber appears to be almost completely coated, thus indicating that the failure most probably occurs at the GO/epoxy interface. This observation is in accordance with the results reported in the following paragraph on the delamination measurements of GO over GF by AFM. It is worthwhile to observe that positive effects on interfacial properties has been also reported for GO coatings electrophorectically deposited onto carbon fibers [42e44]. Short beam shear test was performed to examine the impact of carbon fiber functionalization on the mechanical properties of the carbon fiber/ epoxy resin composites and improvement of $55 \%$ (from 36.7 to $56.9 \mathrm{MPa}$ ) in interlaminar shear strength was reported $[42,43]$.

\subsection{Delamination of GO over GF}

Variable thickness and roughness of GO/GF were monitored with AFM in intermittent contact mode, on topographic images such as that reported in Fig. 8. The bright color shows thicker GO as illustrated from color bar. Four different regions (1, 2, 3 and 4) have been randomly chosen for roughness measurements, and the resulting values reported in Table 4. GO appears not homogenously distributed and thicker region (e.g. 2) where GO is randomly accumulated has significantly higher roughness. 
Delamination of GO from GF was conducted through FFM. It was carried out at the sharp edge interface between GF and GO as shown in a schematic view in Fig. 9a. Black and brown regions represent GF and GO surface respectively. Cantilever probe (slider) follows raster scan pattern in fast scan direction ( $x$-axis) and progress forward in slow scan direction ( $y$-axis) as shows by yellow dashed line. Initially, the surface was scanned with relatively low FN values in order to collect morphological information, in the same region where FFM will be performed. Under low FN the surface elastically recovers, however as FN increases, permanent deformation initiates at some point. Fig. 9b and 9c are FL maps carried out at two different $\mathrm{FN}$ values of $0.753 \mathrm{mN}$ and $3.7 \mathrm{mN}$, respectively. In both cases, FL was recorded due to torsional movement of the cantilever.

The torsional bending of cantilever occurs due to resistance in relative motion offered by $\mathrm{GO}$ and/or GF surfaces. Variable FL is observed at fixed FN due to different angular interactions with tip apex and thickness of GO. Here, we restrict our investigation for a fixed angle (i.e. 90) of interaction between GO and fast scan direction of probe. FL at different angular interactions with sliding tip apex will be considered for future investigations. In Fig. 9b and 9c, bright colors show higher FL obtained at the edges of GF. The quantitative value of $F L$ is plotted in Fig. $9 \mathrm{~d}$ where line profile is carried out from GF to GO covered regions. This picture shows how the cantilever encounters an almost constant lateral force FL along E-glass surface till a point is reached (indicated by a vertical black line) after which FL suddenly increases. Higher FL are required as the cantilever starts piercing GO due to additional force required to peel-off the layers of GO from the GF as well as to destroy the bonds between inter and intra layer of GO. The area under the curve of lateral force displacement plot can be considered as a total dissipated energy (EDISS).

Lateral force ( $F L)$ comprises contribution from glass substrate (FS), from interlayer adhesion between GO layers (FIL) and from interfacial adhesion between GO and GF (FIF), namely:

$$
\mathrm{F}_{\mathrm{L}}=\mathrm{F}_{\mathrm{IL}}+\mathrm{F}_{\mathrm{IF}}+\mathrm{F}_{\mathrm{S}}
$$

FL-FS, removing the contribution from glass friction, contains only contribution from interlayer and interfacial adhesion used to measure the shear strength of the GO/GF interface. FL-FS is measured as $303 \mathrm{nN}$ for current thickness ( $\mathrm{h} \mathrm{z} 22 \mathrm{~nm}$ ) of GO, scratch length (I 1 1 1.45 mm) and width (b 1/4 102 $\mathrm{nm})$. Note that, the aforementioned situation is only possible, 1) when FFM was performed under switch-off feedback loop, otherwise cantilever will follow the topography of GO instead to ploughing; 2) when the substrate (GF) has relatively lower lateral force value than the coating layer (GO). The coefficient of friction (fcof) measured for GF and GO are 0.07 and 0.15 respectively. This favors the delamination of the coating rather than producing a significant wear of the substrate [45]. Fig. 10 reports AFM images of scan size

$2 \mathrm{~mm} 2$ in tapping mode for topography (Fig. 10a) and phase channel (Fig. 10b) show delamination of GO after FFM operations. Fig. 10a indicates that the debris of the GO accumulated at periphery of FFM analyzed region is up to $3 \mathrm{~mm}$ in height. Phase contrast picture of Fig. 10b shows no wear scar on glass fiber and it clearly distinguishes between substrate and delaminated GO. Further, no peeled-off GO was accumulated to the cantilever tip apex. The failure of the interface can be either adhesive or cohesive. In case of former, the failure front propagates strictly at the interface whereas for the latter just below or above the interface within one phase [46]. Generally smaller (than 1) adhesion/cohesive strength ratio lead to "flaking" (adhesive failure) whereas for larger (than 1) values would cause "chipping" (cohesive failure) [47]. Due to the smaller size of scratch width it is difficult to discriminate by the two different mechanisms. At nanoscale Aoyama et al. [48] described abrasion characteristics for adhesive or cohesion failures of thin films (few nanometers) by analyzing the debris collected after scan. In our system for GO film over GF, wear response was characterized as reported in Fig. 10. It shows significant shear displacement of GO along the scanned region and the debris, collected up to $3 \mathrm{~mm}$ (see Fig. 10a, topography), at the periphery of this scanned region. The phase contrast allow us to distinguish between GF and GO debris. This suggests that GO over GF 
exhibited predominant adhesive rather than cohesive failure in our system and under our loading condition (normal force of $3.7 \mathrm{mN}$ ).

Critical normal force $(\mathrm{FN}=3.7 \mathrm{mN}$ ) at which permanent deformation of $\mathrm{GO}$ occurs also produces the measured net shearing force FL-FS. From the work done by this force and from the measurement of the delaminated area we can evaluate the energy dissipated per unit area (2G). Using equation (4) [49] we can derive the shear strength, namely:

$$
2 \Gamma=\frac{E_{\text {DISS }}}{A}=\frac{1}{2} \frac{\left(\tau+\mathrm{Pf}_{\text {cof }}\right)^{2} h}{G_{\mathrm{a}}}
$$

where EDISS is the energy dissipated during delamination under cantilever probe travelled through $1.45 \mathrm{~mm}$ is $4.391013 \mathrm{Nm}, \mathrm{A}$ is the area of delamination, Ga is the shear modulus of the interface, $P$ is the pressure applied and $h$ is the thickness of GO. Assuming the condition of zero applied pressure (i.e. $P=0$ ) in equation (4), shear strength ( $t$ ) can be calculated as follows:

$$
\frac{2 \mathrm{E}_{\mathrm{DISS}}}{1 \mathrm{bh}}=\tau \gamma
$$

where $\mathrm{Ga}=\mathrm{t} / \mathrm{g}$ is the shear modulus of the interface and the shear strain $\mathrm{g}$ can be calculated as $\mathrm{g}=$ $\arctan (\mathrm{y} / \mathrm{h})$; here $\mathrm{y}$ and $\mathrm{h}$ are the elastic shear displacement $(\mathrm{y}=260 \mathrm{~nm})$ and thickness of $\mathrm{GO}(\mathrm{h} \mathrm{z}$ $22 \mathrm{~nm}$ ) respectively. The former one is the critical displacement that can be calculated just before rupture of GO obtained from stick-slip profile at GO edge as determined in Fig. 9d at FN 1/4 0.753 $\mathrm{mN}$. The elastic displacement of GO occurring during interaction with sliding AFM probe with relatively lower FN recovers its original position after removal of shear force, as described by Hunley and coworkers [50]. Accordingly we find $g=1.4$. Thus, the shear strength and hence shear modulus at zero applied pressure are calculated as $\mathrm{t} p=0=1.9108 \mathrm{~N} / \mathrm{m} 2$ and Ga, $\mathrm{p}=0=1.35108 \mathrm{~N} / \mathrm{m} 2$. Substituting this value of $\mathrm{Ga}$ at applied pressure $\mathrm{P}$ z FN/(p r 2 ) $1 / 44.5108 \mathrm{~N} / \mathrm{m} 2$ (where $r$ is the AFM tip radius) in equation (4), $t$ is recalculated as $0.13 \mathrm{GPa}$ which also corresponds to the convergent solution of this iterative method. This value is significantly (about seven times) higher than the ISS values obtained in the single fiber fragmentation measurements (highest value of about $18 \mathrm{MPa}$ ), thus suggesting a failure of the composite at the GO/epoxy rather than at the GO/GF interface, as confirmed by the FESEM observation (see Fig. 9f).

\section{Conclusion}

Negatively charged GO synthesized through modified Hummer's method was stable enough in water suspension and it was deposited on GFs by EPD under the application of different deposition voltages. The amount of GO coating resulted to linearly increase with the deposition voltage.

Fiber-matrix interfacial shear strength evaluated by the fragmentation test on epoxy based microcomposites resulted to increase proportionally to the applied field and a maximum improvement of $219 \%$ was observed. The observed increase in ISS is due to GO coating having functional groups thus making favorable chemical bonding with the epoxy matrix. In addition, AFM tests were performed at nanoscale to delaminate GO over the glass surface of a fiber to measure the adhesion strength between GO and GF. This latter was found to be much higher than the ISS, thus suggesting a failure of the composite at the GO/epoxy rather than at the GO/GF interface and thus proving the efficiency of the fiber coating method here proposed for producing advanced graphene based multiscale composites.

\section{Acknowledgements}


NMP is supported by the European Research Council (ERC StG Ideas 2011 BIHSNAM no. 279985 on "Bio-Inspired Hierarchical Super Nanomaterials", ERC PoC 2015 SILKENE no. 693670 on "Bionic silk with graphene or other nanomaterials spun by silkworms", ERC PoC 2013-II KNOTOUGH no. 632277 on "Super-tough knotted fibres"), by the European Commission under the Graphene Flagship (WP10 "Nanocomposites", no. 604391) and by the Provincia Autonoma di Trento ("Graphene nanocomposites", no. S116/ 2012e242637 and reg. delib. no. 2266).

\section{References}

[1] Lintel Report. Growth Opportunities in Global Composites Industry 2014- 2019, Texas (USA): Lucintel, Dallas, 2014.

[2] D. Pedrazzoli, A. Pegoretti, Silica nanoparticles as coupling agents for polypropylene/glass composites, Compos. Sci. Technol. 76 (4) (2013) 77e83.

[3] A. Pegoretti, J. Karger-Kocsis, Interphase engineering in polymer composites: Challenging the devil..., Express Polym. Lett. 9 (10) (2015) 838.

[4] F.R. Jones, A Review of Interphase Formation and Design in Fibre-Reinforced Composites, J. Adhes. Sci. Technol. 24 (1) (2010) 171e202.

[5] J. Karger-Kocsis, H. Mahmood, A. Pegoretti, Recent advances on fiber/matrix interphase engineering in polymer composites, Prog. Mater. Sci. 73 (2015) 1 e43.

[6] L. Brely, F. Bosia, N.M. Pugno, A Hierarchical Lattice Spring Model to Simulate the Mechanics of 2-D Materials-Based Composites, Front. Mater. 2 (2015).

[7] H. Qian, E.S. Greenhalgh, M.S.P. Shaffer, A. Bismarck, Carbon nanotube-based hierarchical composites: a review, J. Mater. Chem. 20 (23) (2010) 4751 e4762.

[8] Z. Shen, S. Bateman, D.Y. Wu, P. McMahon, M. Dell'Olio, J. Gotama, The effects of carbon nanotubes on mechanical and thermal properties of woven glass fibre reinforced polyamide- 6 nanocomposites, Compos. Sci. Technol. 69 (2) (2009) $239 \mathrm{e} 244$.

[9] E.T. Thostenson, W.Z. Li, D.Z. Wang, Z.F. Ren, T.W. Chou, Carbon nanotube/ carbon fiber hybrid multiscale composites, J. Appl. Phys. 91 (9) (2002) 6034e6037.

[10] X. He, F. Zhang, R. Wang, W. Liu, Preparation of a carbon nanotube/carbon fiber multi-scale reinforcement by grafting multi-walled carbon nanotubes onto the fibers, Carbon 45 (13) (2007) $2559 \mathrm{e} 2563$.

[11] L. Mei, X. He, Y. Li, R. Wang, C. Wang, Q. Peng, Grafting carbon nanotubes onto carbon fiber by use of dendrimers, Mater. Lett. 64 (22) (2010) $2505 \mathrm{e} 2508$.

[12] E. Bekyarova, E.T. Thostenson, A. Yu, H. Kim, J. Gao, J. Tang, et al., Multiscale Carbon NanotubeCarbon Fiber Reinforcement for Advanced Epoxy Composites, Langmuir 23 (7) (2007) 3970 e3974.

[13] X. Wang, H. Liu, P. Fang, L. Liao, C. Pan, K.M. Liew, Interface Enhancement of Glass Fiber/Vinyl Ester Composites with Carbon Nanotubes Synthesized from Ethanol Flames, J. Nanosci. Nanotechnol. 10 (2) (2010) 948e955.

[14] J. Zhang, R. Zhuang, J. Liu, E. Mader, G. Heinrich, S. Gao, Functional interphases $€$ with multiwalled carbon nanotubes in glass fibre/epoxy composites, Carbon 48 (8) (2010) 2273 e2281. 
[15] J.K. Park, I.-H. Do, P. Askeland, L.T. Drzal, Electrodeposition of exfoliated graphite nanoplatelets onto carbon fibers and properties of their epoxy composites, Compos. Sci. Technol. 68 (7e8) (2008) 1734e1741.

[16] A.C. Ferrari, F. Bonaccorso, V. Fal'ko, K.S. Novoselov, S. Roche, P. Boggild, et al., Science and technology roadmap for graphene, related two-dimensional crystals, and hybrid systems, Nanoscale 7 (11) (2015) 4598e4810.

[17] K.S. Novoselov, A.K. Geim, S.V. Morozov, D. Jiang, Y. Zhang, S.V. Dubonos, et al., Electric Field Effect in Atomically Thin Carbon Films, Science 306 (5696) (2004) 666e669.

[18] C. Lee, X. Wei, J.W. Kysar, J. Hone, Measurement of the Elastic Properties and Intrinsic Strength of Monolayer Graphene, Science 321 (5887) (2008) 385e388.

[19] A.A. Balandin, S. Ghosh, W. Bao, I. Calizo, D. Teweldebrhan, F. Miao, et al., Superior Thermal Conductivity of Single-Layer Graphene, Nano Lett. 8 (3) (2008) 902e907.

[20] K.S. Hu, D.D. Kulkarni, I. Choi, V.V. Tsukruk, Graphene-polymer nanocomposites for structural and functional applications, Prog. Polym. Sci. 39 (11) (2014) 1934e1972.

[21] D. Pedrazzoli, A. Pegoretti, Hybridization of short glass fiber polypropylene composites with nanosilica and graphite nanoplatelets, J. Reinf. Plastics Compos. 33 (18) (2014) 1682e1695.

[22] D. Pedrazzoli, A. Pegoretti, Expanded graphite nanoplatelets as coupling agents in glass fiber reinforced polypropylene composites, Compos. Part A Appl. Sci. Manuf. 66 (2014) 25e34.

[23] D. Pedrazzoli, A. Pegoretti, K. Kalaitzidou, Understanding the effect of silica nanoparticles and exfoliated graphite nanoplatelets on the crystallization behavior of isotactic polypropylene, Polym. Eng. Sci. 55 (3) (2015) 672e680.

[24] J.N. Israelachvili, Intermolecular and Surface Forces, revised third ed., Academic press, 2011.

[25] A.W. Adamson, A.P. Gast, Physical chemistry of surfaces, 1967.

[26] L.Y. Jiang, Y. Huang, H. Jiang, G. Ravichandran, H. Gao, K.C. Hwang, et al., A cohesive law for carbon nanotube/polymer interfaces based on the van der Waals force, J. Mech. Phys. Sol 54 (11) (2006) $2436 \mathrm{e} 2452$.

[27] B. Shen, W. Zhai, C. Chen, D. Lu, J. Wang, W. Zheng, Melt Blending In situ Enhances the Interaction between Polystyrene and Graphene through pep Stacking, ACS Appl. Mater. interfac. 3 (8) (2011) 3103e3109.

[28] H.-L. Zhang, X.-L. Wei, Y. Zang, J.-Y. Cao, S. Liu, X.-P. He, et al., Fluorogenic Probing of Specific Recognitions between Sugar Ligands and Glycoprotein Receptors on Cancer Cells by an Economic Graphene Nanocomposite, Adv. Mater. 25 (30) (2013) 4097e4101.

[29] S. Pei, H.-M. Cheng, The reduction of graphene oxide, Carbon 50 (9) (2012) 3210e3228.

[30] Q. Cheng, M. Wu, M. Li, L. Jiang, Z. Tang, Ultratough Artificial Nacre Based on Conjugated Cross-linked Graphene Oxide, Angew. Chem. 125 (13) (2013) 3838e3843.

[31] D.D. Kulkarni, I. Choi, S.S. Singamaneni, V.V. Tsukruk, Graphene OxidePolyelectrolyte Nanomembranes, ACS Nano 4 (8) (2010) 4667e4676.

[32] D. Pedrazzoli, A. Pegoretti, K. Kalaitzidou, Synergistic effect of exfoliated graphite nanoplatelets and short glass fiber on the mechanical and interfacial properties of epoxy composites, Compos. Sci. Technol. 98 (2014) 15e21. 
[33] W.S. Hummers, R.E. Offeman, Preparation of Graphitic Oxide, J. Am. Chem. Soc. 80 (6) (1958), $1339 \mathrm{e} 1339$.

[34] W. Tyson, A. Kelly, Tensile properties of fibre-reinforced metals: copper/ tungsten and copper/molybdenum, J. Mech. Phys. Sol 13 (6) (1965) 329e338.

[35] M.R. Gurvich, A.T. Dibenedetto, A. Pegoretti, Evaluation of the statistical parameters of a Weibull distribution, J. Mater. Sci. 32 (14) (1997) 3711e3716.

[36] C.P. Green, H. Lioe, J.P. Cleveland, R. Proksch, P. Mulvaney, J.E. Sader, Normal and torsional spring constants of atomic force microscope cantilevers, Rev. Sci. Instrum. 75 (6) (2004) 1988.

[37] J.E. Sader, J.W.M. Chon, P. Mulvaney, Calibration of rectangular atomic force microscope cantilevers, Rev. Sci. Instrum. 70 (10) (1999) 3967.

[38] S. Das, D. Lahiri, A. Agarwal, W. Choi, Interfacial bonding characteristics between graphene and dielectric substrates, Nanotechnology 25 (4) (2014), 045707.

[39] Q. An, A.N. Rider, E.T. Thostenson, Hierarchical composite structures prepared by electrophoretic deposition of carbon nanotubes onto glass fibers, ACS Appl. Mater. interfac. 5 (6) (2013) 2022e2032.

[40] A. Lerf, H. He, M. Forster, J. Klinowski, Structure of graphite oxide revisited, J. Phys. Chem. B 102 (23) (1998) 4477e4482.

[41] J. Chen, D. Zhao, X. Jin, C. Wang, D. Wang, H. Ge, Modifying glass fibers with graphene oxide: Towards high-performance polymer composites, Compos. Sci. Technol. 97 (0) (2014) 41e45.

[42] C. Deng, J. Jiang, F. Liu, L. Fang, J. Wang, D. Li, et al., Influence of graphene oxide coatings on carbon fiber by ultrasonically assisted electrophoretic deposition on its composite interfacial property, Surf. coat Technol. 272 (2015) 176e181.

[43] C. Deng, J. Jiang, F. Liu, L. Fang, J. Wang, D. Li, et al., Effects of electrophoretically deposited graphene oxide coatings on interfacial properties of carbon fiber composite, J. Mater. Sci. 50 (17) (2015) 5886e5892.

[44] S.-Y. Huang, G.-P. Wu, C.-M. Chen, Y. Yang, S.-C. Zhang, C.-X. Lu, Electrophoretic deposition and thermal annealing of a graphene oxide thin film on carbon fiber surfaces, Carbon 52 (2013) 613 e616.

[45] K. Lee, A. Polycarpou, Shear strength determination using the nanoscratch technique and its application to thin solid films, J. Mater. Res. 21 (2006) 2304e2313.

[46] F. Creuzet, G. Ryschenkow, H. Arribart, A new tool for adhesion science: the atomic force microscope, J. adhes 40 (1) (1992) $15 \mathrm{e} 25$.

[47] H.E. Hintermann, Thin solid films to combat friction, wear, and corrosion, J. Vac. Sci. Technol. B Microelectron. Nanom. Struct. 2 (4) (1984) 816.

[48] S. Aoyama, Y.T. Park, C.W. Macosko, T. Ougizawa, G. Haugstad, AFM probing of polymer/nanofiller interfacial adhesion and its correlation with bulk mechanical properties in a poly(ethylene terephthalate) nanocomposite, Langmuir 30 (43) (2014) 12950e12959.

[49] N.M. Pugno, Q. Yin, X. Shi, R. Capozza, A generalization of the Coulomb's friction law: from graphene to macroscale, Meccanica 48 (8) (2013) 1845e1851.

[50] D.P. Hunley, T.J. Flynn, T. Dodson, A. Sundararajan, M.J. Boland, D.R. Strachan, Friction, adhesion, and elasticity of graphene edges, Phys. Rev. B (3) (2013) 87, 035417. 
Table 1

Physical properties of epoxy resin.

\begin{tabular}{ll}
\hline Physical property & Value \\
\hline Glass transition temperature $\left(\mathrm{T}_{\mathrm{g}}\right)$ & $28^{\circ} \mathrm{C}$ \\
Thermal degradation & $340{ }^{\circ} \mathrm{C}$ \\
Yield stress $\left(\sigma_{\mathrm{y}}\right)$ & $22.8 \pm 2.4 \mathrm{MPa}$ \\
Tensile strength $\left(\sigma_{\mathrm{T}}\right)$ & $26.1 \pm 1.1 \mathrm{MPa}$ \\
Young's Modulus & $795 \pm 28 \mathrm{MPa}$ \\
\hline
\end{tabular}

Table 2

Mechanical properties of GF as determined from single fiber tensile tests. ( $\mathrm{N}=$ number of specimens, $\bar{R}=$ average strength at $\mathrm{L}=20 \mathrm{~mm}, \sigma_{0}=$ scale parameter at $\mathrm{L}_{0}=5 \mathrm{~mm}, \mathrm{~m}=$ shape parameter, $\nu=$ coefficient of variation).

\begin{tabular}{ll}
\hline Parameter & Value \\
\hline $\mathrm{N}$ & 31 \\
$\bar{R}$ & $1007 \mathrm{MPa}$ \\
$\sigma_{0}$ & $1476 \mathrm{MPa}$ \\
$\mathrm{m}$ & 4.4 \\
$\nu$ & $28.2 \%$ \\
\hline
\end{tabular}

Table 3

Effect of EPD on the fragmentation tests results.

\begin{tabular}{|c|c|c|c|c|}
\hline $\begin{array}{l}\text { Applied } \\
\text { voltage } \\
(\mathrm{V} / \mathrm{cm})\end{array}$ & $\begin{array}{l}\text { Fragments length at saturation } \mathrm{L}_{\mathrm{s}} \\
(\mathrm{mm})\end{array}$ & $\begin{array}{l}\text { Critical length } L_{c} \\
(\mathrm{~mm})\end{array}$ & $\begin{array}{l}\text { Tensile strength of fiber at the critical length } \sigma_{\mathrm{fb}}\left(\mathrm{L}_{\mathrm{c}}\right) \\
\text { (MPa) }\end{array}$ & $\begin{array}{l}\text { Interfacial shear strength ISS } \\
\text { (MPa) }\end{array}$ \\
\hline 0 (uncoated) & $2.65 \pm 0.9$ & $3.53 \pm 1.2$ & $1475 \pm 111$ & $5.7 \pm 2.2$ \\
\hline 2.5 & $2.00 \pm 0.2$ & $2.70 \pm 0.2$ & $1546 \pm 27$ & $7.0 \pm 0.7$ \\
\hline 5 & $1.72 \pm 0.3$ & $2.30 \pm 0.4$ & $1611 \pm 69$ & $8.9 \pm 2.1$ \\
\hline 7.5 & $1.21 \pm 0.1$ & $1.61 \pm 0.1$ & $1742 \pm 35$ & $14.6 \pm 1.5$ \\
\hline 10 & $0.94 \pm 0.1$ & $1.26 \pm 0.1$ & $1844 \pm 47$ & $18.2 \pm 2.5$ \\
\hline
\end{tabular}

Table 4

Roughness measurement of four marked locations.

\begin{tabular}{lcc}
\hline $\begin{array}{l}\text { Marked areas } \\
\text { number }\end{array}$ & $\begin{array}{l}\text { Roughness average Ra } \\
(\mathrm{nm})\end{array}$ & Root mean square RMS (nm) \\
\hline 1 & 8.216 & 10.72 \\
2 & 58.300 & 20.05 \\
3 & 3.198 & 4.04 \\
4 & 2.797 & 3.56 \\
\hline
\end{tabular}




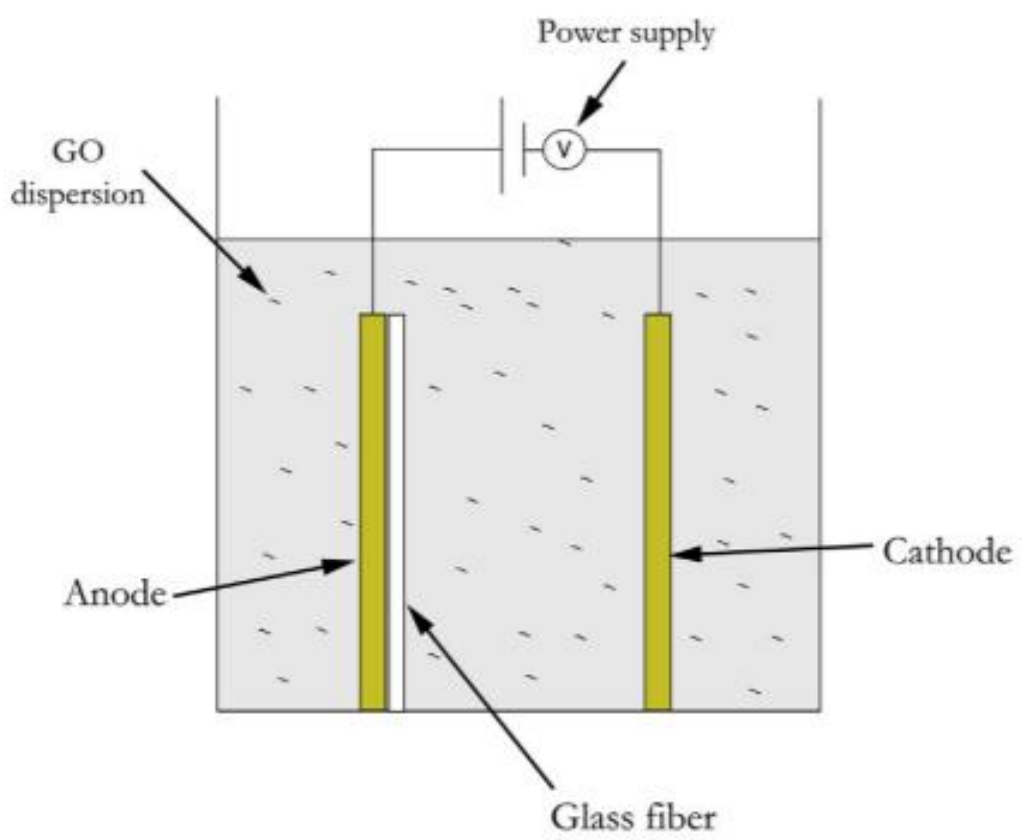

Fig. 1. Schematics of the electrophoretic deposition process of GO on GF.

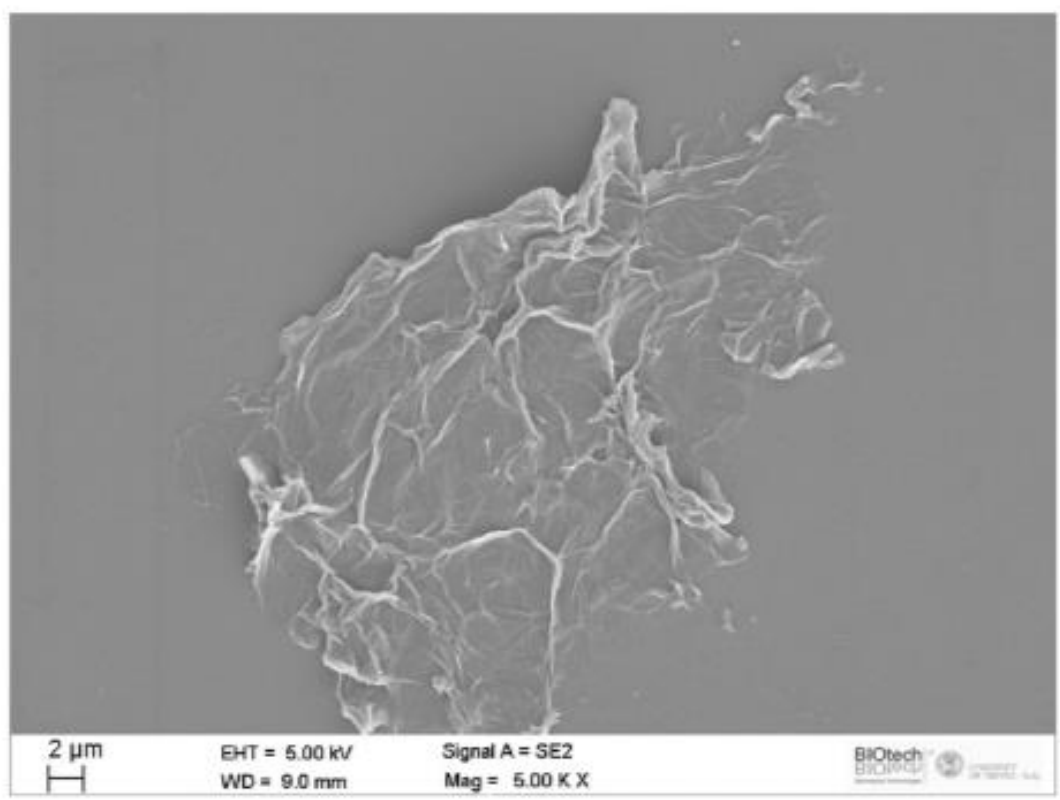

Fig. 2. FESEM image of a GO nanosheet. 


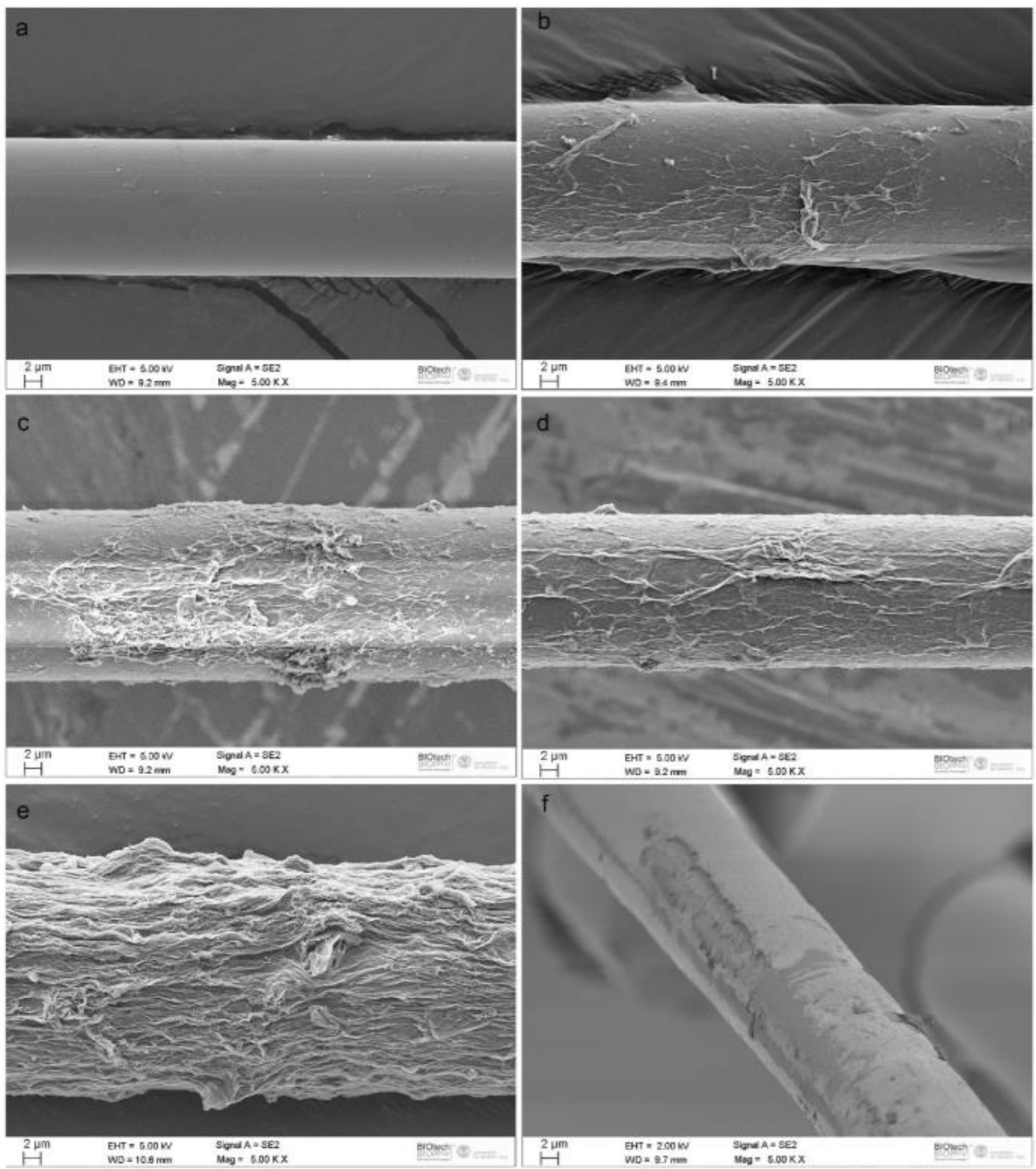

Fig. 3. FESEM images of GFs a) uncoated and coated with GO under a deposition field of b) $2.5 \mathrm{~V} / \mathrm{cm}, \mathrm{c}) 5 \mathrm{~V} / \mathrm{cm}$, d) $7.5 \mathrm{~V} / \mathrm{cm}$, e) $10 \mathrm{~V} / \mathrm{cm}$. f) debonded fiber after tensile failure of microcomposite with GO-coated GFs under a deposition field of $10 \mathrm{~V} / \mathrm{cm}$. 


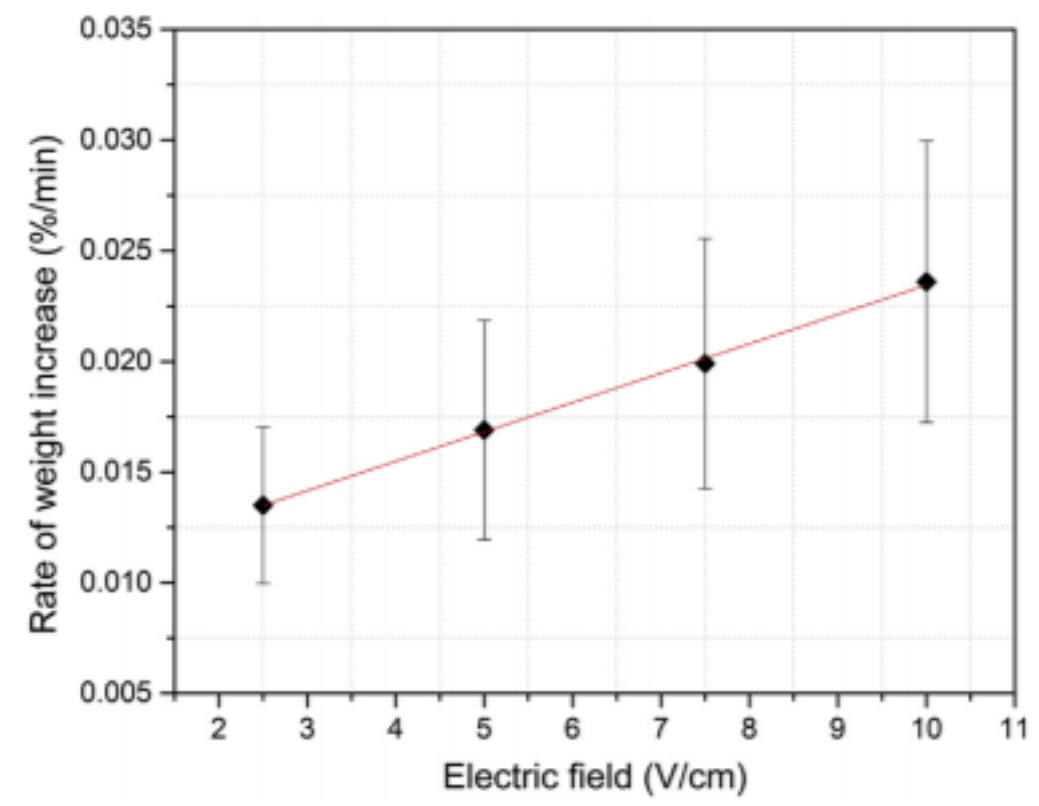

Fig. 4. Rate of weight increase of GO coated glass fiber as a function of the applied field during EPD process.

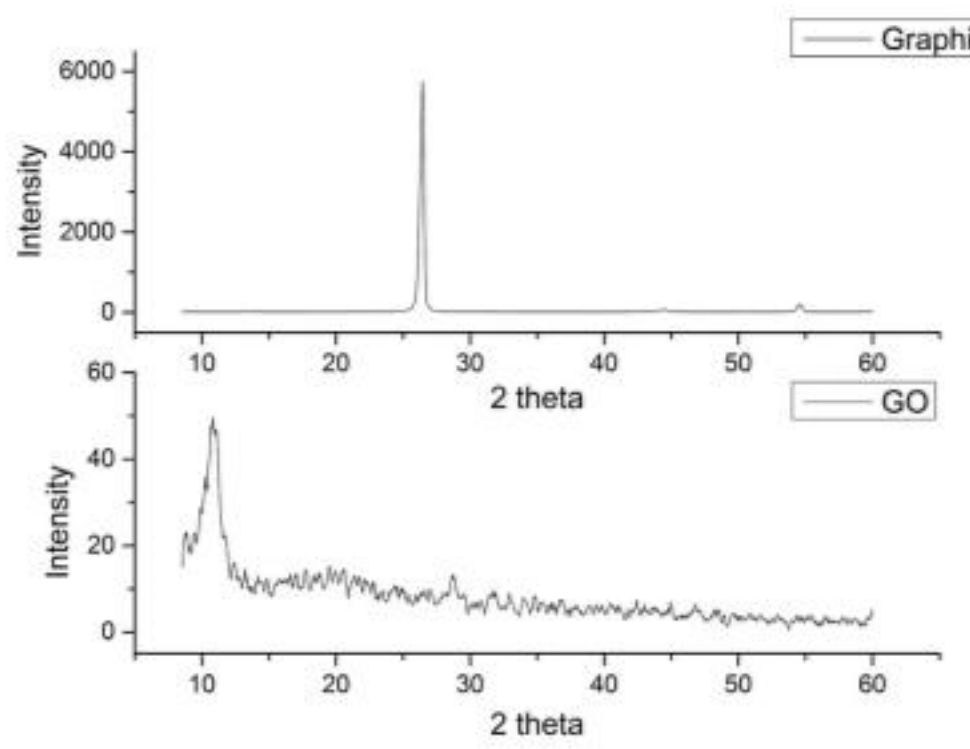

Fig. 5. XRD diffractograms of pristine graphite and GO. 


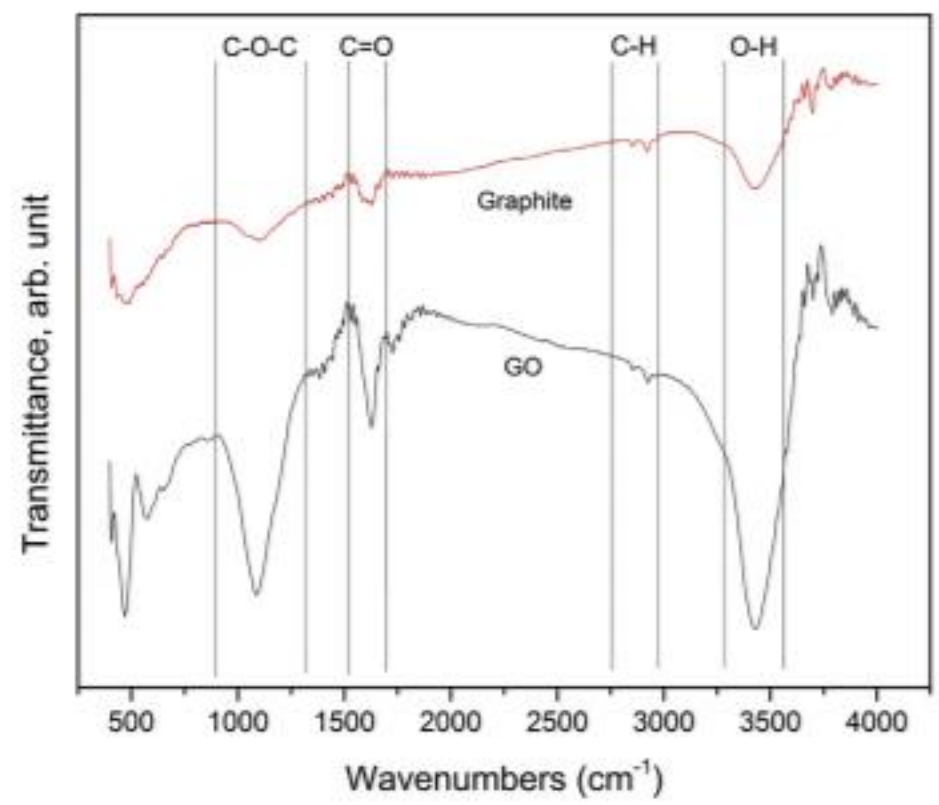

Fig. 6. FMR spectra of pristine graphite and GO.

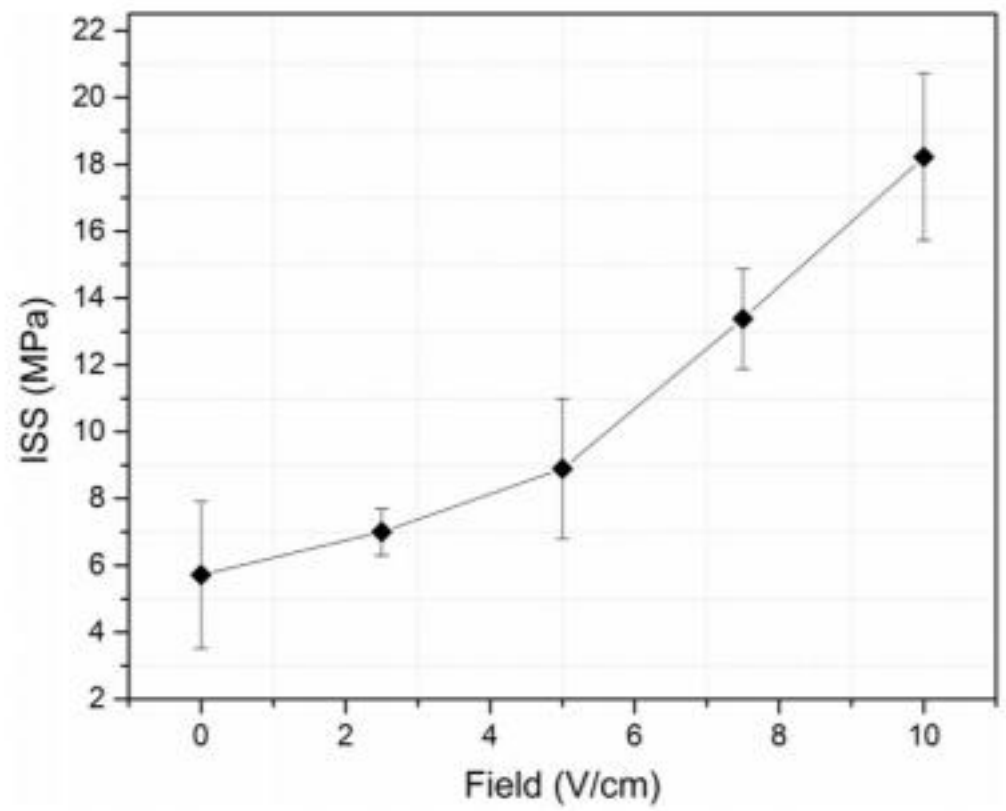

Fig. 7. Fiber-matrix interfacial shear strength values as determined by the fragmentation test on epoxy microcomposites containing E-glass fiber coated with GO. The point at $0 \mathrm{~V} / \mathrm{cm}$ refer to bare fibers without GO coating. 
$1.86 \mu \mathrm{m}$

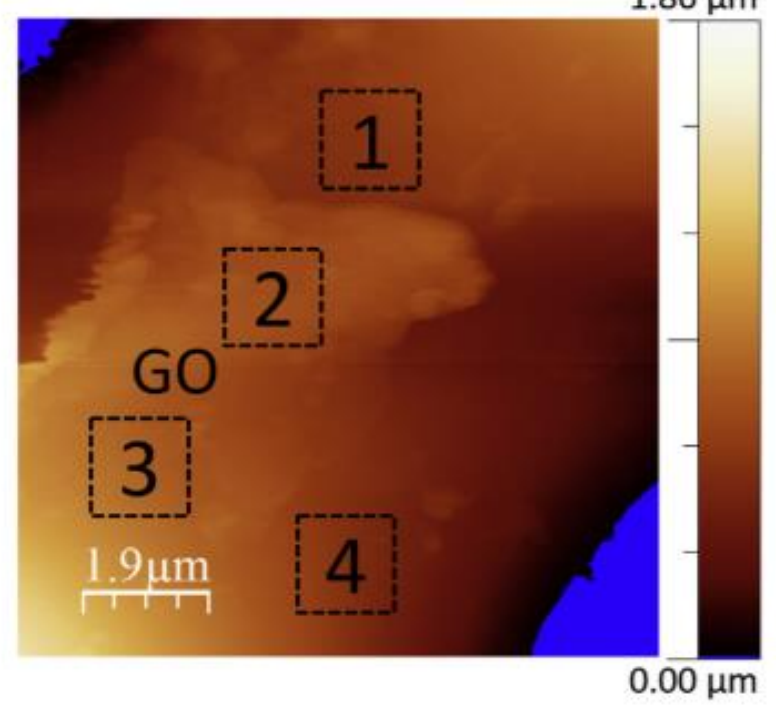

Fig. 8. Topographic AFM image (height channel) shows GO on glass, scale bar shows different thickness of GO. Regions $1-4$ are randomly chosen $\left(1.5 \mu \mathrm{m}^{2}\right)$ to carry out roughness measurements as given in Table 4. 


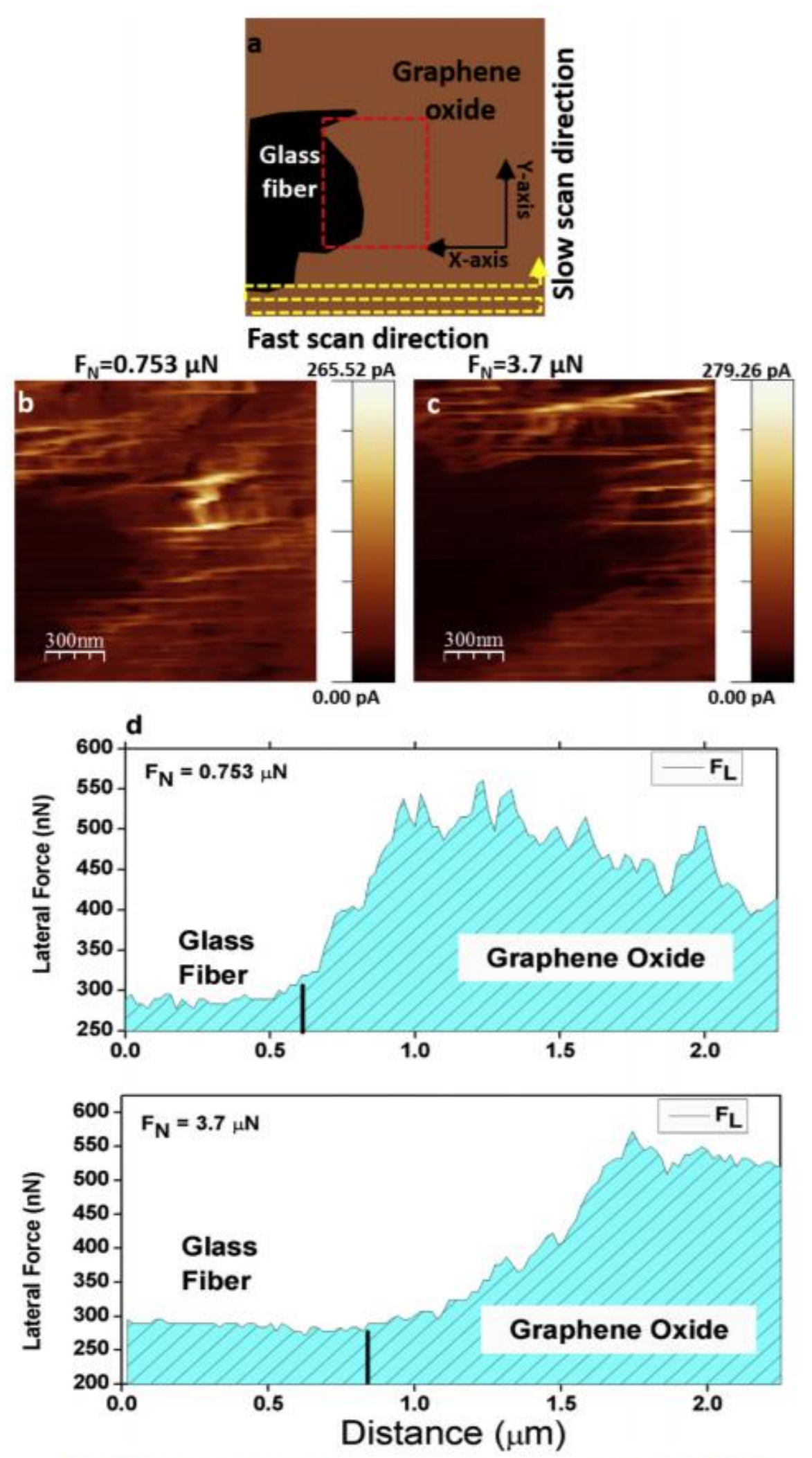

Fig. 9. a) Schematic view of glass substrate (black color) and Go covered glass regions (brown) mimics the $F_{2}$ map given in b) and c). Yellow dashed line shows raster scanning pattern of AFM probe in $X$ and $Y$ directions, b) and $c$ ) show $F_{2}$ map of same regions at different $F_{N}$ where $G 0$ oxide start delaminating in $c$ ) at critical $F_{N}$. d) shows lateral force displacement plot carried out at $\mathrm{F}_{\mathrm{N}}$. 


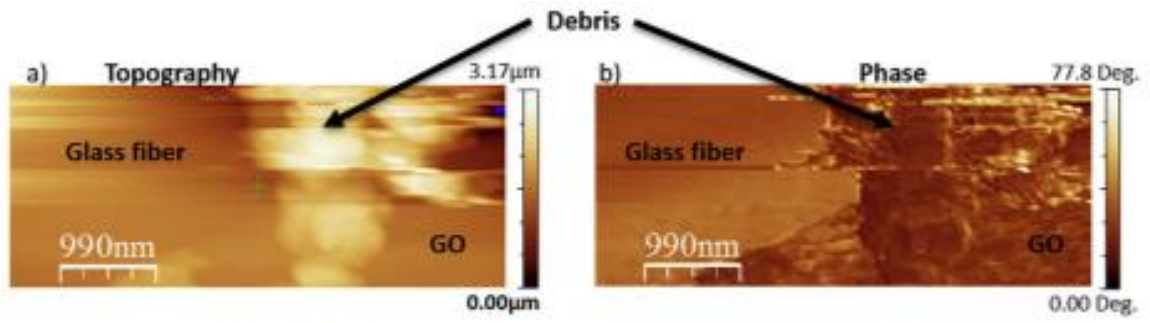

Fig. 10. AFM image of scan size $5 \times 2 \mu \mathrm{m}^{2}$ in tapping mode for topography (a) and phase channel (b) shows delamination of Go after FFM operations. 